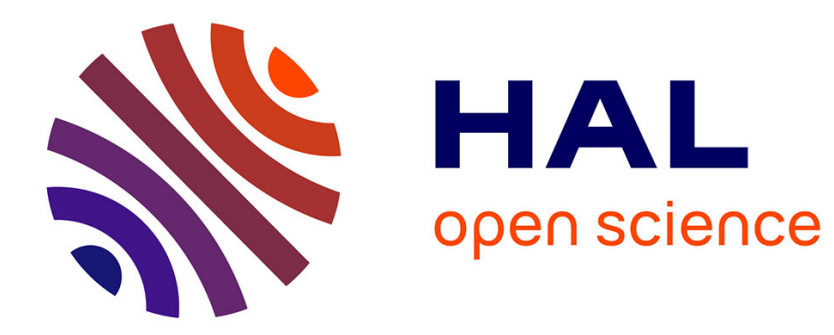

\title{
From Crowd Simulation to Robot Navigation in Crowds
}

Thierry Fraichard, Valentin Levesy

\section{To cite this version:}

Thierry Fraichard, Valentin Levesy. From Crowd Simulation to Robot Navigation in Crowds. IEEE Robotics and Automation Letters, 2020, 5 (2), pp.729-735. 10.1109/LRA.2020.2965032 . hal02461493

\section{HAL Id: hal-02461493 \\ https://hal.inria.fr/hal-02461493}

Submitted on 30 Jan 2020

HAL is a multi-disciplinary open access archive for the deposit and dissemination of scientific research documents, whether they are published or not. The documents may come from teaching and research institutions in France or abroad, or from public or private research centers.
L'archive ouverte pluridisciplinaire HAL, est destinée au dépôt et à la diffusion de documents scientifiques de niveau recherche, publiés ou non, émanant des établissements d'enseignement et de recherche français ou étrangers, des laboratoires publics ou privés. 


\title{
From Crowd Simulation to Robot Navigation in Crowds
}

\author{
Thierry Fraichard ${ }^{\dagger}$, and Valentin Levesy ${ }^{\dagger}$
}

\begin{abstract}
This letter presents the result of a study aiming at investigating to what extent the results obtained in the Crowd Simulation domain could be used to control a mobile robot navigating among people. It turns out that Crowd Simulation relies on two assumptions that would not hold for a real mobile robot, a test protocol has therefore been designed in order to thoroughly evaluate how three representative Crowd Simulation techniques would perform when said assumptions are relaxed. The study shows that all those techniques entail safety problems, i.e. they would cause collisions in the real world. The study also highlights the most promising candidate for a transposition on a real mobile robot.
\end{abstract}

Index Terms-Crowd Simulation; Autonomous Navigation; Human-Robot Motion;

\section{INTRODUCTION}

\section{A. Background and Motivation}

T HE late 90s saw the deployment of the first autonomous mobile robots in environments featuring people. In 1997, the RHINO robot spent six days guiding visitors at the Deutsches Museum in Bonn [1]. A year later, the Minerva robot moved through crowds during two weeks at the Smithsonian's National Museum of American History in Washington, USA [2] and the MAid wheelchair robot was tested in the central station of Ulm during rush hour and in the Hannover Messe' 98 in Germany for 36 hours total [3]. In 2002, eleven Robox mobile robots operated daily for six months at the Swiss national exhibition Expo.02 [4].

Despite these early achievements and the growing number of research efforts on robot navigation in crowds, e.g. [5], [6], [7], [8], [9], it must be acknowledged that mobile robots casually navigating in crowds are not a reality yet. This may seem surprising given that self-driving cars can routinely be seen driving on highways and roads among heavy traffic sometimes. In both cases, i.e. road networks and crowds, the robot has to deal with highly uncertain dynamic environments. The most likely explanation is that human-populated environments are not as structured as the road network and that people's behavior is not governed by explicit traffic rules. As a consequence, robots navigating in crowds suffer from the wellknown Freezing Robot Problem [10], i.e. when the density of the crowd increases, the robot freezes on the spot because it cannot find a forward motion deemed safe enough with respect to people's highly uncertain behavior. It is therefore unable to jostle its way through the crowd.

Manuscript received: September 6, 2019; Revised November 22, 2019; Accepted December 21, 2019. This paper was recommended for publication by Editor Allison M. Okamura upon evaluation of the Associate Editor and Reviewers' comments. This work was partially supported by the French Ministry of Research and the LIG project C-NA-2019-EMERGENCE17.

${ }^{\dagger}$ Univ. Grenoble Alpes, INRIA, CNRS, Grenoble INP, LIG, F-38000 Grenoble, France.

Digital Object Identifier (DOI): 10.1109/LRA.2020.2965032.
In contrast, the scientific domain of Crowd Simulation, which was born in the late 80s [11], has by now produced a lot of techniques that allow a virtual agent to easily navigate among high-density virtual crowds featuring hundreds of people ( $c f$. \&II $)$. A "crowd simulation" search on Internet produces a long list of impressive videos. The result of this search begs the following question: how come what is seemingly achieved so easily by a virtual agent cannot be achieved by a real robot? The main of the research presented in this paper is to try to answer this question by investigating to what extent the results obtained in Crowd Simulation could be transposed to the realm of robot navigation in crowds.

\section{B. Contributions}

The first step was to review the main Crowd Simulation (CS) techniques from the literature and to examine them carefully from a Robotics perspective. This examination has revealed three issues that are problematic.

The first issue has to do with the nature of the crowd. To the best of the authors' knowledge, all CS techniques assume that the virtual agents composing the crowd have the same behavior. In other words, they are all controlled by the same algorithm. It is obvious that this assumption, henceforth called the Homogeneity assumption, does not hold in the real world. The behavior of a person in a crowd is determined by several factors, e.g. goal, mood, etc., and those factors are unique to each person.

The second issue has to do with the information that is provided to the virtual agent about its environment. A large number of CS techniques assume that each virtual agent has a perfect knowledge of its surroundings including the shape, position, and velocity of all the static and moving obstacles around. Again, this assumption, henceforth called the Omniscience assumption, does not hold for a real robot which has to rely on a set of limited on-board sensors to gather information about its surroundings.

The third and last issue has to do with Safety. The primary objective of CS is to compute visually realistic crowd simulation. Should collisions and minor intersections between virtual agents happen, it would not bo a problem as long as it remained unnoticeable to the observer. Such collisions are of course unacceptable for a real robot.

The second step was to put a selection of representative CS techniques to the test and see how they performed when both the Homogeneity and the Omniscient assumptions were relaxed. To that end, an experimental protocol was devised and three performance criteria were chosen to evaluate the CS techniques considered. The first two performance criteria are standards from a Robotics perspective, i.e. safety and efficiency. The third one has to do with the human-like nature 
of the motions generated by the CS techniques. Indeed, a number of studies has shown that people prefer when robots behave in a human-like fashion, e.g. [12], [13], [14], [15].

Finally, the results of the test campaign are presented, analyzed, and discussed. The tests reveal that a CS technique that works well in simulation will not perform that well if implemented on a real mobile robot, safety being the most important issue. Of the two CS assumptions, the tests also shows that the Homogeneity assumption is the most critical. Although the study does not provide a clear-cut answer as to which CS technique should be selected in order to be adapted on a real mobile robot, it helps decide which one is the best candidate among the representative CS techniques considered in the study.

\section{Outline of the paper}

The paper is organized as follows: the CS literature is reviewed in $\$ \mathrm{II}$. The CS techniques selected for evaluation purposes are presented in III The evaluation protocol is detailed in $\$ \mathrm{IV}$. The experimental results are presented and analyzed in $\mathrm{V}$.

\section{Crowd Simulation Overview}

The Encyclopedia of Computer Graphics and Games defines Crowd Simulation (CS) as the process of simulating the movement and/or the behavior of a large number of entities or characters [16]. The simulated characters are expected to move to their goals, interact with their environment, and respond to each other. CS has many applications such as urban planning [17], evacuation simulation [18], and of course animating characters in video games and movies.

There are two fundamentally distinct philosophies to simulating crowds: the macroscopic approach and the microscopic approach. Macroscopic approaches, e.g. [19], [20], [21], treat the crowd as a continuum medium characterized by averaged quantities such as density and mean velocity; the whereas microscopic approaches distinguish the individuals composing the crowd: each individual is an autonomous virtual agent whose behavior is controlled by its own navigation strategy. It remains to be seen if macroscopic approaches could be adapted to control actual mobile robots. Microscopic approaches are more relevant in the sense that the navigation strategy of each virtual agent could in principle be transferred to an actual mobile robot. CS has been an active domain since the pioneering work of [11] and a lot of microscopic approaches have been proposed over the years. Even though they are very different, the review of the literature shows that three important categories have emerged: force-based, velocity-based, and vision-based.

\section{A. Force-Based Approaches}

These approaches consider each agent as a particle subject to various interaction forces. These forces can be exerted by the environment, e.g. repulsion from obstacles, or by internal motivations of the agent, e.g. joining a group. The net force determines the final motion of the agent. The Social Force
Model proposed in [22] is one of the most influential models in this category. It has inspired several extensions, e.g. [23], [24], [25], [26].

\section{B. Velocity-Based Approaches}

These approaches operates on the velocity space of the agent and seek to compute collision-free motion over a short time horizon, e.g. [27], [28], [29], [30]. To that end, short term prediction of the other agents' future motions are used.

\section{Vision-Based Approaches}

Acknowledging the importance of human visual information on the regulation of human locomotion [31], vision-based approaches seek to reproduce the role of vision on human locomotion. To that end, visual cues, e.g. optical flow, time to collision, and focus of expansion, are computed and used to control the agent, e.g. [32], [33], [34], [35]

However different these CS techniques may appear, they all assume that the virtual agents composing the crowd are controlled by the same algorithm. Interestingly enough, this Homogeneity assumption is never questioned. As far as forcebased and velocity-based approaches are concerned, they assume that each virtual agent has a perfect knowledge of its surroundings including the shape, position, and velocity of all the static and moving obstacles around. This is an Omniscience assumption of sorts. Vision-based approaches are, by design, not concerned by this assumption. It is also important to note that the main objective of CS is to produce simulations that are visually pleasant and realistic. To the extent that if collisions happen between the agents, it only becomes a problem if they cause "visually unpleasant artifacts" to quote from [20]. As mentioned earlier, these two assumptions and collisions are problematic from a Robotics standpoint.

\section{Selected CRowd Simulation Techniques}

Given the plethora of microscopic CS techniques, it is of course impossible to put them all to the test in order to check what happens when the Homogeneity and Omniscience assumptions are relaxed. It was decided to chose one representative technique for each category identified in $\$$ II They are briefly presented below, and the reader is referred to the articles cited for a full presentation.

\section{A. Universal Power Law (UPL)}

UPL $^{1}[24]$ is a force-based approach. The crowd is modeled as an interacting particle systems. UPL is an extension of the well-known Social-Force Model (SFM) [22]. Among SFM-based techniques, UPL has been selected because the interaction forces between the agents are not defined in an ad-hoc and somewhat arbitrary manner; they are derived from the analysis of a large collection of human motion data.

\footnotetext{
${ }^{1}$ http://motion.cs.umn.edu/PowerLaw
} 


\section{B. Reciprocal Velocity Obstacles (RVO)}

$\mathrm{RVO}^{2}[36$ is a velocity-based approach. It was originally developed to address multi-robot collision avoidance problems. It builds upon the well-known concept of Velocity Obstacles [37]. Besides its popularity, RVO has been selected because it provides a sufficient condition for each agent to avoid collision over a given time horizon assuming that the other agents also use RVO.

\section{Vision-Based Navigation (VBN)}

VBN [34] is a vision-based approach. Each agent is equipped with a synthetic vision component that computes two visual cues, i.e. the distance and time of closest approaches with an other agent, that have been shown in [38] to be relevant in the regulation of human locomotion. These cues are used to estimate a collision risk which is then minimized by the control strategy. VBN has been selected because it has been designed so as to decrease the collision risk.

All three approaches feature a nominal (or preferred) walking speed for the agents that will be selected by the approach if it belongs to the set of admissible speeds.

\section{Experimental Protocol and Performance CRITERIA}

The homogeneity and omniscience assumptions having been identified as being problematic from a Robotics perspective, it was decided to test the candidate CS techniques in situations where these assumptions were relaxed. The need for controllable and reproducible test conditions led to the use of a simulated environment ( $\$$ IV-A). Five test scenarios have been defined ( $(\mathrm{IV}-\mathrm{B})$, and three performance criteria have been chosen for evaluation purposes ( $\$ \mathrm{IV}-\mathrm{C})$.

\section{A. Simulation Software}

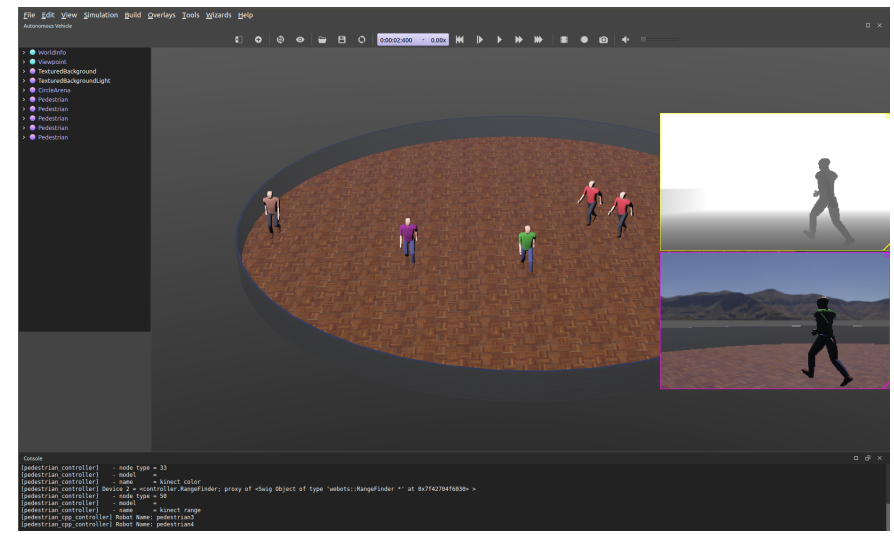

Figure 1: Webots and ROS-based virtual world simulator.

The Webots $3^{3}$ simulation software and the $\operatorname{ROS}^{4}$ (Robot Operating System) framework have been used to define virtual environments within which virtual agents, pedestrian or mobile

\footnotetext{
${ }^{2}$ http://gamma.cs.unc.edu/RVO2

3 https://www.cyberbotics.com

4 http://www.ros.org
}

robots, can be individually defined with their own geometry, kinematics, dynamics and sensing characteristics. Each virtual agent is controlled by its own navigation strategy. Fig. 1] shows the user interface of the system, it displays the arena where the agents moves and the output of a virtual RGB-D sensor mounted on one of the agent that can be used to simulate the visual perception of the agent. The three CS techniques UPL, RVO and VBN have been implemented from scratch in C++.

\section{B. Test Scenarios}
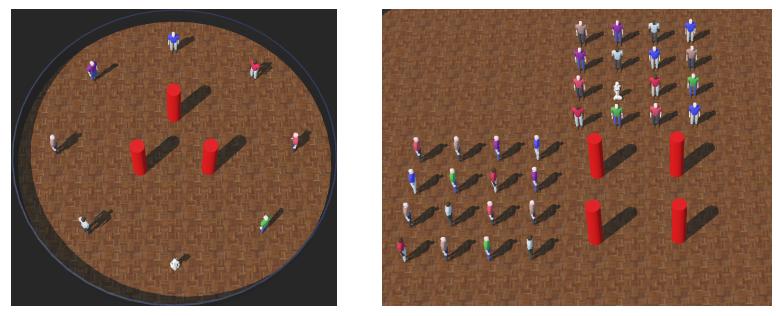

Figure 2: Circle and Crossing scenarios.
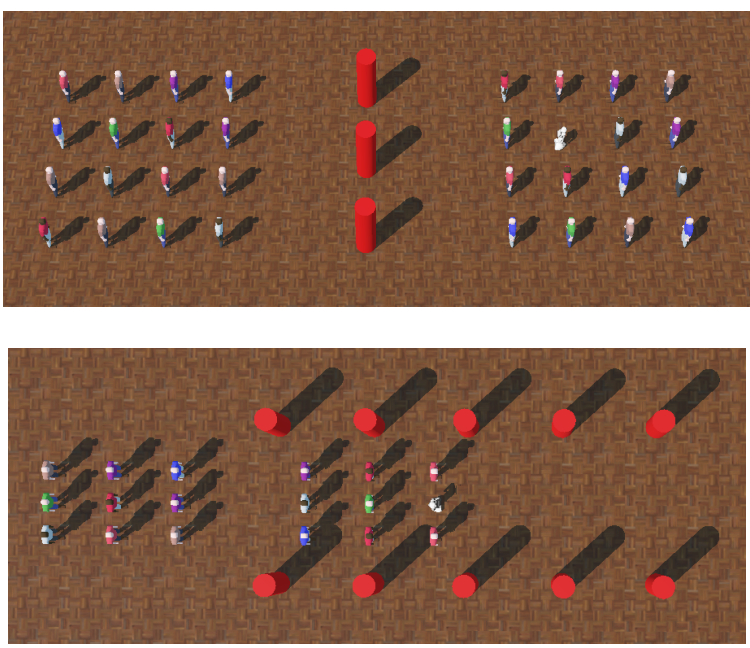

Figure 3: Opposite and Overtake scenarios.

The first three scenarios are standard benchmarks of CS (Figs. 2 and 3 . They are respectively called Circle (8 agents placed on a circle have to reach their antipodal positions), Crossing (two groups of 16 agents moving forward with a $90^{\circ}$ crossing angle) and Opposite (two groups of 16 agents moving in opposite directions). Two variants of these scenarios are considered: with and without fixed obstacles (red pillars). A fourth scenario called Overtake has been added (Fig. 3), it features two groups of 9 agents moving in the same direction in a corridor formed by pillars, the group behind moving faster than the other. In those four scenarios, each agent is assigned a goal and the simulation stops after 40s or when every agent has reached its goal (whichever comes first). A final scenario called Random was added to simulate unorganized crowds (Fig. 11). It features 22 agents who are assigned a sequence of random goal positions within a circular arena. In this case, the simulation runs for $1 \mathrm{~min}$.

In all scenarios (except Overtake), the agents are assigned a nominal speed that obeys the normal distribution $\mathcal{N}(1.5,0.1)$ 
in order to simulate the variations that can be found in a crowd ( $1.5 \mathrm{~m} / \mathrm{s}$ being close to the average human walking speed [39]). In the Overtake scenario, the slow (resp. fast) group has a nominal speed obeying $\mathcal{N}(0.5,0.1)$ (resp. $\mathcal{N}(2.0,0.1)$ ).

\section{Performance Criteria}

As pointed out by [40], the objective evaluation of a CS technique is not a simple task, a single and commonly accepted performance measure does not exist. Three performance criteria have been chosen, the first two are standard from a Robotics perspective, i.e. safety and efficiency. The third one has to do with the human-like nature of the motions generated.

1) Safety: the safety of a CS technique is measured by simply counting the number of collisions it yields. When an agent collides with a fixed obstacle, it takes full responsibility for the collision, i.e. collision count +1 . When two agents collide, the responsibility is equally shared between them, i.e. collision count +0.5 .

2) Efficiency: suppose an agent has to move from point $A$ to point $B$ at a given nominal speed $v_{n o m}$ and that it reaches $B$ at time $t_{f}$ using a given CS technique. The efficiency of the CS technique is defined as follows:

$$
\frac{d(A, B)}{t_{f} v_{n o m}}
$$

where $d(A, B)$ denotes the Euclidean distance between $A$ and $B$. Eq.(1) is the ratio of the "straight line speed" to the nominal speed, it is best when close to 1 .

3) Human-Like Behavior: as mentioned earlier, people prefer when robots behave in a human-like fashion, it is therefore desirable that a CS technique yields motions that appear to be human-like. Because this criterion is nearly impossible to define in a quantitative manner, it is resorted to the subjective examination of the shape of the motions 5 and the speed distribution during the motions in order to identify aberrant results.

\section{EXPERIMENTAL RESUlts AND ANALYSIS}

\section{A. Relaxing the Homogeneity Assumption}

The objective of the first series of experiments carried out is to evaluate the impact on the performances of the three candidate CS techniques when the Homogeneity assumption is relaxed. To that end, the 8 scenarios considered are run 10 times for RVO, UPL and VBN under the Homogeneity assumption, i.e. with all agents using the same CS technique. Then the Homogeneity assumption is relaxed and each agent in a given scenario is assigned a random CS technique uniformly selected among RVO, UPL or VBN. Again, the 8 scenarios considered are run 10 times. Tables $\mathrm{I}$ and $\mathrm{II}$ respectively report the results of this series of experiments for both the safety and efficiency criteria 6

From a safety standpoint (table I), two surprising things have been revealed by this series of experiments. First, RVO

\footnotetext{
${ }^{5}$ Note that this is what is usually done in CS works.

${ }^{6}$ For all tables, the standard deviation of the tests were small enough to have no significant effects on the \% difference reporting.
}

which is the only CS technique that provides a form of collision avoidance guarantee under the Homogeneity assumption turns out to be the one whose safety performance drops dramatically when this assumption is relaxed: over $400 \%$ more collisions on average. Second, both VBN and UPL performs better when the Homogeneity assumption is relaxed: over $30 \%$ less collisions. Now, although this observation seems bad news for RVO, it is important to also observe that RVO is the technique that has by far the best safety record: consider the last line of table I] the average number of collisions per agent for RVO is much better than those of VBN and UPL, even in the heterogeneous case. VBN is the worst in this respect.

From an efficiency standpoint (table. II), the differences between the homogeneous and the heterogeneous cases are not that significant: from $9 \%$ increase to $7 \%$ decrease of efficiency. Besides, as shown in the last line of table III. all techniques have a similar efficiency record whatever the situation.
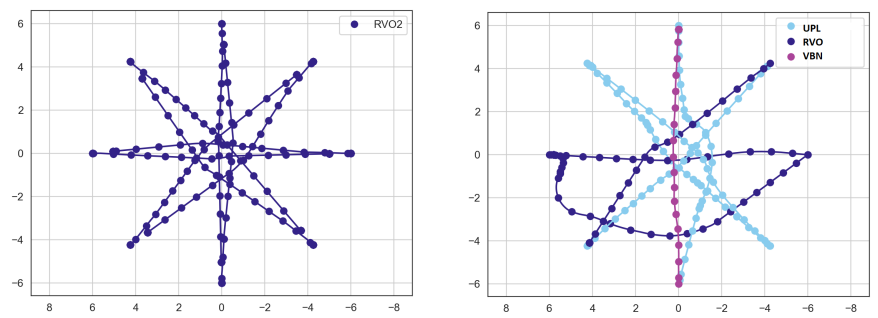

Figure 4: Circle scenario, homogeneous RVO case (top) vs. heterogeneous case (bottom).

As far as evaluating whether the CS techniques yield human-like motions, it is a trickier issue. The behaviors observed between the homogeneous and the heterogeneous cases were not significantly different except for RVO where aberrant behaviors could sometimes be observed in the heterogeneous case: see for instance the situation depicted in Fig. 4 . In the heterogeneous case, the aberrant behavior of the RVOcontrolled agent starting from the left and moving to the right can easily be observed.

Besides, the analysis of the speed distributions for the three $\mathrm{CS}$ techniques in both the homogeneous and heterogeneous cases also revealed an unexpected feature of RVO compared to UPL and VBN: an agent driven by RVO will spend significantly more time at a speed slower than the nominal speed. This is illustrated in Fig. 5 that depicts the speed distributions for the CS techniques in the Crossing scenario. In each plot, the vertical axis is the amount of time spent by all agents at the corresponding speed. Note how both UPL and VPN distributions are unimodal with one peak around the nominal speed whereas the RVO distribution is clearly bimodal with a second peak at a much slower speed. In the heterogeneous case (Fig. 5d), it can be observed that this effect is increased, the RVO distribution flattens (as well as UPL but to a lesser extent).

\section{B. Relaxing the Omniscience Assumption}

The objective of the second series of experiments carried out is to evaluate the impact on the performances of RVO 


\begin{tabular}{l|c|c|c|c|c|c|c|c|c}
\hline \multirow{2}{*}{ Scenario } & \multicolumn{3}{|c|}{ RVO } & \multicolumn{3}{c|}{ VBN } & \multicolumn{3}{c}{ UPL } \\
\cline { 2 - 9 } & Homo. & Hetero. & \% Diff. & Homo. & Hetero. & \% Diff. & Homo. & Hetero. & \% Diff. \\
\hline Circle & 0.00 & 0.00 & 0.00 & 0.00 & 0.00 & 0.00 & 0.05 & 0.00 & -100.00 \\
Circle + obstacles & 0.00 & 0.00 & 0.00 & 0.01 & 0.07 & 432.80 & 0.01 & 0.03 & 100.00 \\
Opposite & 0.00 & 0.01 & 267.65 & 0.03 & 0.03 & 32.00 & 0.06 & 0.00 & -100.00 \\
Opposite + obstacles & 0.01 & 0.05 & 455.56 & 0.05 & 0.16 & 201.89 & 0.15 & 0.07 & -51.72 \\
Crossing & 0.01 & 0.23 & 1740.00 & 1.65 & 0.93 & -43.64 & 0.56 & 0.38 & -32.14 \\
Crossing + obstacles & 0.02 & 0.28 & 1397.33 & 1.35 & 0.83 & -38.52 & 0.58 & 0.40 & -30.80 \\
Overtake & 0.01 & 0.04 & 260.36 & 0.22 & 0.11 & -51.85 & 0.40 & 0.08 & -80.85 \\
Random & 0.20 & 0.70 & 250.00 & 2.75 & 1.88 & -31.64 & 1.19 & 0.85 & -28.57 \\
\hline Total & 0.03 & 0.16 & 424.00 & 0.76 & 0.50 & -33.83 & 0.37 & 0.23 & -39.67
\end{tabular}

Table I: Relaxing the Homogeneity assumption from a safety standpoint. For each scenario and each CS technique is reported the average number of collisions caused per agent and per run in the homogeneous and heterogeneous cases. \% Diff. is the evolution in percentage. The last line presents the consolidated results. Possible discrepancies are due to truncation.

\begin{tabular}{l|c|c|c|c|c|c|c|c|c}
\hline \multirow{2}{*}{ Scenario } & \multicolumn{3}{|c|}{ RVO } & \multicolumn{3}{c|}{ VBN } & \multicolumn{3}{c}{ UPL } \\
\cline { 2 - 9 } & Homo. & Hetero. & \% Diff. & Homo. & Hetero. & \% Diff. & Homo. & Hetero. & \% Diff. \\
\hline Circle & 0.99 & 0.99 & 0.68 & 0.96 & 0.86 & -10.42 & 0.70 & 0.87 & 23.81 \\
Circle + obstacles & 0.72 & 0.87 & 20.19 & 0.79 & 0.77 & -3.36 & 0.68 & 0.71 & 4.90 \\
Opposite & 0.77 & 0.77 & -0.17 & 0.78 & 0.74 & -5.31 & 0.59 & 0.73 & 23.73 \\
Opposite + obstacles & 0.70 & 0.65 & -7.11 & 0.79 & 0.67 & -15.97 & 0.60 & 0.67 & 12.22 \\
Crossing & 0.56 & 0.61 & 8.46 & 0.57 & 0.53 & -6.98 & 0.62 & 0.66 & 5.66 \\
Crossing + obstacles & 0.47 & 0.52 & 11.43 & 0.61 & 0.59 & -4.35 & 0.60 & 0.68 & 12.58 \\
Overtake & 0.47 & 0.60 & 27.71 & 0.59 & 0.57 & -4.06 & 0.61 & 0.55 & -9.98 \\
Random & 0.32 & 0.47 & 43.97 & 0.61 & 0.56 & -9.11 & 0.63 & 0.63 & -0.74 \\
\hline Total & 0.63 & 0.68 & 9.60 & 0.71 & 0.66 & -7.19 & 0.63 & 0.69 & 9.34
\end{tabular}

Table II: Relaxing the Homogeneity assumption from an efficiency standpoint. For each scenario and each CS technique is reported the average efficiency per agent and per run in the homogeneous and heterogeneous cases. \% Diff. is the evolution in percentage. The last line presents the consolidated results. Possible discrepancies are due to truncation.

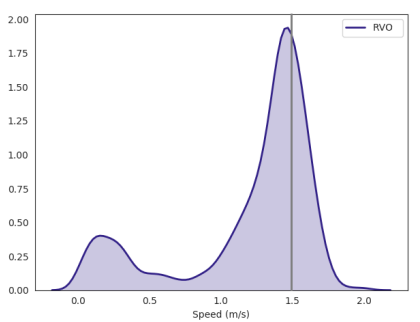

(a) RVO

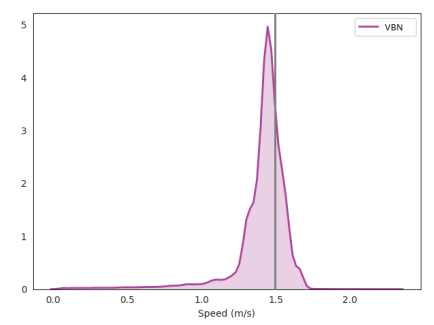

(c) VBN

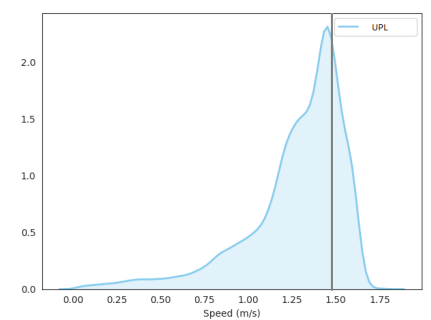

(b) UPL

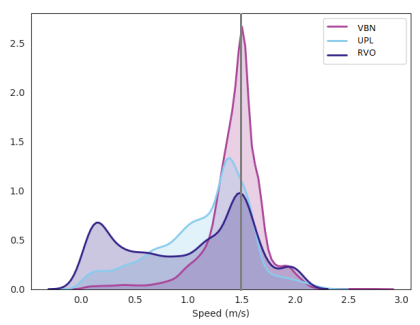

(d) Heterogeneous case

Figure 5: Speed distributions during a run of the Crossing scenario (nominal speed $=1.5 \mathrm{~m} / \mathrm{s}$, vertical gray line). Homogeneous $(\mathrm{a}, \mathrm{b}, \mathrm{c}) v s$. heterogeneous cases $(\mathrm{d})$.

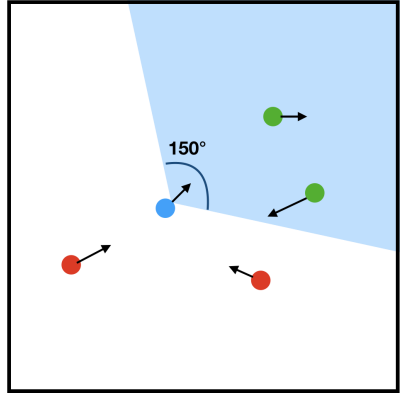

Figure 6: $150^{\circ}$ horizontal field-of-view.

and UPL when the Omniscience assumption is relaxed (VPN is not concerned here since it assumes that each agent has a sensor-based perception of the environment). To that end, the 9 scenarios considered are run 10 times for RVO and UPL under the Omniscience assumption, i.e. with all agents having perfect knowledge of their surroundings. Then the Omniscience assumption is relaxed and each agent now has a limited sensor-based perception of its environment, i.e. each agent has a $150^{\circ}$ horizontal field-of-view similar to the one used in VBN (Fig. 6). Again, the 9 scenarios considered are run 10 times. Tables $[\mathrm{III}]$ and $\mathrm{IV}$ report the results of this series of experiments for both the safety and efficiency criteria.

From a safety standpoint, the performance of UPL slightly 


\begin{tabular}{l|c|c|c|c|c|c}
\hline \multirow{2}{*}{ Scenario } & \multicolumn{3}{|c|}{ UPL } & \multicolumn{3}{c}{ RVO } \\
\cline { 2 - 7 } & Omni. & Unomni. & $\%$ Diff. & Omni. & Unomni. & $\%$ Diff. \\
\hline Circle & 0.050 & 0.075 & 50.00 & 0.000 & 0.000 & 0.00 \\
Circle + obstacles & 0.013 & 0.013 & 0.00 & 0.000 & 0.000 & 0.00 \\
Opposite & 0.058 & 0.040 & -31.03 & 0.003 & 0.000 & -100.00 \\
Opposite + obstacles & 0.145 & 0.120 & -17.24 & 0.009 & 0.006 & -33.33 \\
Crossing & 0.560 & 0.570 & 1.78 & 0.013 & 0.018 & 44.00 \\
Crossing + obstacles & 0.578 & 0.640 & 10.72 & 0.019 & 0.025 & 33.69 \\
Overtake & 0.400 & 0.550 & 37.50 & 0.011 & 0.011 & 0.00 \\
Random & 0.518 & 0.570 & 10.04 & 0.233 & 0.130 & -44.21 \\
\hline Total & 0.290 & 0.322 & 11.03 & 0.036 & 0.024 & -33.33
\end{tabular}

Table III: Relaxing the Omniscience assumption from a safety standpoint. For each scenario and each CS technique is reported the average number of collisions caused per agent and per run in the omniscient and unomniscient cases. \% Diff. is the evolution in percentage. The last line presents the consolidated results. Possible discrepancies are due to truncation.

\begin{tabular}{l|c|c|c|c|c|c}
\hline \multirow{2}{*}{ Scenario } & \multicolumn{3}{|c|}{ UPL } & \multicolumn{3}{c}{ RVO } \\
\cline { 2 - 7 } & Omni. & Unomni. & \% Diff. & Omni. & Unomni. & \% Diff. \\
\hline Circle & 0.70 & 0.72 & 2.86 & 0.99 & 0.97 & -1.35 \\
Circle + obstacles & 0.68 & 0.65 & -4.31 & 0.72 & 0.76 & 5.74 \\
Opposite & 0.59 & 0.60 & 1.24 & 0.77 & 0.77 & -0.17 \\
Opposite + obstacles & 0.60 & 0.63 & 4.44 & 0.70 & 0.74 & 5.78 \\
Crossing & 0.62 & 0.60 & -3.31 & 0.56 & 0.54 & -3.46 \\
Crossing + obstacles & 0.60 & 0.57 & -5.08 & 0.47 & 0.47 & 1.43 \\
Overtake & 0.61 & 0.62 & 0.87 & 0.47 & 0.48 & 3.86 \\
Random & 0.63 & 0.72 & 12.63 & 0.32 & 0.33 & 2.91 \\
\hline Total & 0.63 & 0.64 & 1.59 & 0.63 & 0.64 & 1.59
\end{tabular}

Table IV: Relaxing the Omniscience assumption from an efficiency standpoint. For each scenario and each CS technique is reported the average efficiency per agent and per run in the omniscient and unomniscient cases. \% Diff. is the evolution in percentage. The last line presents the consolidated results. Possible discrepancies are due to truncation.

decreases, $11 \%$ more collisions, while that of RVO increases, $33 \%$ less collisions.

From an efficiency standpoint, the differences between the omniscient and the unomniscient cases are not that significant: around $1.5 \%$ increase of efficiency for both UPL and RVO.

From a human-like behavior standpoint, unnatural oscillatory behaviors were observed for RVO in the unomniscient case. They appear as soon as an obstacle is near the limit of the field-of-view. In such a situation with, for example, an obstacle on the right side limit of the field-of-view of the agent, the agent would turn left to avoid collision with the obstacle. Said obstacle would then disappear from the fieldof-view and the agent would turn right to resume moving toward its goal. By doing so, the obstacle would reappear in the field-of-view. The repetition of this process produces the aforementioned oscillations. It is interesting to note that the first RVO approach [29] was developed in order to address this oscillation issue that affects several Robotics collision avoidance techniques.

\section{Analysis of the Results}

The first lesson of the test campaign reported here is the impact of relaxing the Homogeneity assumption from a safety standpoint. What is maybe the most surprising result is the fact that RVO, a very popular collision avoidance technique in Robotics, is the one that is the most negatively impacted: $>400 \%$ additional collisions observed. It is a bit ironic since RVO is the only technique that provides a form of safety guarantee when the Homogeneity assumption holds. It is also surprising to observe that both VBN and UPL perform better. This somewhat counter-intuitive result could be explained by the fact that VPN and UPL were confronted to RVO which does much better in terms of collision avoidance. From an efficiency standpoint, relaxing the Homogeneity assumption has no significant impact on all techniques although the analysis revealed what could be considered a weak point of RVO, namely the bimodal nature of its speed distribution.

Contrary to what might have been expected, relaxing the Omniscience assumption improves the safety performance of RVO while having no significant impact on the safety performance of UPL. From an efficiency standpoint, the Omniscience assumption has no significant impact on UPL and RVO.

\section{CONClusion AND Future Works}

The purpose of the study reported here was to investigate to what extent a CS technique could be used to control a mobile robot navigating among people. To that end, three representative CS techniques, i.e. UPL, RVO and VBN, were put to the test and their performance evaluated when the 
Homogeneity and Omniscience assumptions were relaxed (as would be the case for a real mobile robot).

The study does not provide a clear cut answer as to which CS technique should be selected in order to be adapted on a real mobile robot. All techniques have a safety problem, they would yield collisions in the real world. VBN is clearly the worst candidate in this respect, RVO and UPL are close with a slight advantage to RVO. However, RVO has been shown to be prone to aberrant behaviors when the Homogeneity assumption is relaxed. Besides it suffers from a questionable speed distribution that adversely affects its efficiency. For these two reasons, UPL appears to be the best choice. It will have to be adapted in order to control a real mobile robot and modified so as to deal with the safety issue. One possibility is to turn it into a passively safe navigation strategy in the manner of [41]. Of course, tests in the real world will be necessary to demonstrate the ability of the strategy to navigate among real people.

\section{REFERENCES}

[1] W. Burgard, A. Cremers et al., "The Museum Tour-Guide Robot RHINO," in Autonome Mobile Systeme, ser. Informatik aktuell, H. Wörn, R. Dillmann et al., Eds. Springer, 1998.

[2] S. Thrun, M. Beetz et al., "Probabilistic Algorithms and the Interactive Museum Tour-Guide Robot Minerva," Int. Journal of Robotics Research, vol. 19, no. 11, pp. 972-999, Nov. 2000.

[3] E. Prassler, J. Scholz et al., "Navigating a Robotic Wheelchair in a Railway Station during Rush Hour," Int. Journal of Robotics Research, vol. 18, no. 7, pp. 711-727, Jul. 1999.

[4] R. Siegwart, K. Arras et al., "Robox at Expo.02: A large-scale installation of personal robots," Robotics and Autonomous Systems, vol. 42, no. 3, pp. 203-222, Mar. 2003.

[5] G. Ferrer, A. Garrell et al., "Robot companion: A social-force based approach with human awareness-navigation in crowded environments," in IEEE/RSJ Int. Conf. Intelligent Robots and Systems (IROS), Tokyo (JP), Nov. 2013.

[6] P. Trautman, J. Ma et al., "Robot navigation in dense human crowds: Statistical models and experimental studies of human-robot cooperation," Int. Journal of Robotics Research, vol. 34, no. 3, pp. 335-356, 2015.

[7] M. Chen, Y. an Everett, M. Liu et al., "Socially aware motion planning with deep reinforcement learning," in IEEE/RSJ Int. Conf. on Intelligent Robots and Systems (IROS), Vancouver (CA), Sep. 2017.

[8] Y. Luo, P. Cai et al., "PORCA: Modeling and Planning for Autonomous Driving Among Many Pedestrians," IEEE Robotics and Automation Letters, vol. 3, no. 4, pp. 3418-3425, Oct. 2018.

[9] T. Fan, X. Cheng et al., "Getting robots unfrozen and unlost in dense pedestrian crowds," IEEE Robotics and Automation Letters, vol. 4, no. 2, pp. 1178-1185, Apr. 2019

[10] P. Trautman and A. Krause, "Unfreezing the robot: Navigation in dense, interacting crowds," in IEEE/RSJ Int. Conf. on Intelligent Robots and Systems (IROS), Taipei (TW), Oct. 2010.

[11] C. Reynolds, "Flocks, herds and schools: A distributed behavioral model," Comput. Graph., vol. 21, no. 4, pp. 25-34, Jul. 1987.

[12] C. Lichtenthäler, T. Lorenzy et al., "Influence of legibility on perceived safety in a virtual human-robot path crossing task," in IEEE Int. Symp. on Robot and Human Interactive Communication (RO-MAN), Paris (FR), Sep. 2012.

[13] D. Carton, W. Olszowy et al., "Measuring the effectiveness of readability for mobile robot locomotion," Int. Journal of Social Robotics, vol. 5 , 2016.

[14] C. Vassallo, A.-H. Olivier et al., "How do walkers avoid a mobile robot crossing their way?" Gait \& Posture, vol. 51, pp. 97-103, Jan. 2017.

[15] - "How do walkers behave when crossing the way of a mobile robot that replicates human interaction rules?" Gait \& Posture, vol. 60, pp. 188-193, Feb. 2018.

[16] D. Thalman, "Crowd simulation," in Encyclopedia of Computer Graphics and Games, N. Lee, Ed. Springer, Feb. 2016.
[17] G. Drettakis, M. Roussou et al., "Design and evaluation of a real-world virtual environment for architecture and urban planning," Presence: Teleoperators and Virtual Environments, vol. 16, no. 3, pp. 318-332, 2007.

[18] S. Gwynne, E. Galea et al., "A review of the methodologies used in the computer simulation of evacuation from the built environment," Building and Environment, vol. 34, no. 6, pp. 741-749, Nov. 1999.

[19] R. Hughes, "A continuum theory for the flow of pedestrians," Transportation Research Part B: Methodological, vol. 36, no. 6, pp. 507-535, Jul. 2002.

[20] A. Treuille, S. Cooper et al., "Continuum crowds," ACM Transactions on Graphics, vol. 25, no. 3, pp. 1160-1168, Jul. 2006.

[21] M. Twarogowska, P. Goatin et al., "Macroscopic modeling and simulations of room evacuation," Applied Mathematical Modelling, vol. 38, no. 24, pp. 5781-5795, Dec. 2014.

[22] D. Helbing and P. Molnár, "Social force model for pedestrian dynamics," Physical Review E, vol. 51, no. 5, pp. 4282-4286, May 1995.

[23] T. Lakoba, D. Kaup et al., "Modifications of the helbing-molnár-farkasvicsek social force model for pedestrian evolution," Simulation, vol. 81, no. 5, pp. 339-352, May 2005.

[24] I. Karamouzas, B. Skinner et al., "Universal power law governing pedestrian interactions," Physical Review Letters, vol. 113, no. 23, Dec. 2014.

[25] X. Zhang, X. Zhang et al., "Extended social force model-based mean shift for pedestrian tracking under obstacle avoidance," IET Computer Vision, vol. 11, no. 1, pp. 1-9, 2017.

[26] X. Truong and T. Ngo, "Toward socially aware robot navigation in dynamic and crowded environments: A proactive social motion model," IEEE Trans. on Automation Science and Engineering, vol. 14, no. 4, pp. $1743-1760$, Oct. 2017.

[27] W. Shao and D. Terzopoulos, "Animating autonomous pedestrians," in ACM SIGGRAPH 2005 Sketches, 2005, p. 45.

[28] S. Paris, J. Pettré et al., "Pedestrian reactive navigation for crowd simulation: a predictive approach," Computer Graphics Forum, vol. 26, no. 3, pp. 665-674, 2007.

[29] J. Van den Berg, M. Lin et al., "Reciprocal velocity obstacles for real-time multi-agent navigation," in IEEE Int. Conf. on Robotics and Automation (ICRA), Pasadena (US), May 2008.

[30] I. Karamouzas, P. Heil et al., "A predictive collision avoidance model for pedestrian simulation," in Motion in Games, ser. Lecture Notes in Computer Science, A. Egges, R. Geraerts et al., Eds. Springer, 2009, vol. 5854, pp. 41-52.

[31] A. Patla, "Understanding the roles of vision in the control of human locomotion," Gait \& Posture, vol. 5, no. 1, pp. 54-69, Feb. 1997.

[32] D. Terzopoulos and T. Rabie, "Animat vision: Active vision in artificial animals," in IEEE Int. Conf. Computer Vision, Cambridge (US), Jun. 1995.

[33] J. Ondrej, J. Pettre et al., "A synthetic-vision based steering approach for crowd simulation," ACM Transactions on Graphics, vol. 29, no. 4, Jul. 2010.

[34] T. Dutra, R. Marques et al., "Gradient-based steering for vision-based crowd simulation algorithms," Computer Graphics Forum, vol. 36, no. 2, pp. 337-348, May 2017.

[35] A. Lòpez, F. Chaumette et al., "Character navigation in dynamic environments based on optical flow," Computer Graphics Forum, vol. 38 , no. 2, pp. 181-192, May 2019.

[36] J. Van den Berg, S. Guy et al., "Reciprocal n-body collision avoidance," in Robotics Research, ser. Springer Tracts in Advanced Robotics (STAR), C. Pradalier, R. Siegwart et al., Eds., 2011, vol. 70, pp. 3-19.

[37] P. Fiorini and Z. Shiller, "Motion planning in dynamic environments using velocity obstacles," Int. Journal of Robotics Research, vol. 17, no. 7, pp. 760-772, 1998.

[38] R. Meerhoff, J. Bruneau et al., "Guided by gaze: Prioritization strategy when navigating through a virtual crowd can be assessed through gaze activity," Acta Psychologica, vol. 190, pp. 248-257, Oct. 2018.

[39] R. Levine and A. Norenzayan, "The pace of life in 31 countries," Journal of Cross-Cultural Psychology, vol. 30, no. 2, pp. 178-205, Mar. 1999.

[40] D. Wolinski, S. Guy et al., "Parameter estimation and comparative evaluation of crowd simulations," Computer Graphics Forum, vol. 33, no. 2, pp. 303-312, May 2014.

[41] S. Bouraine, T. Fraichard et al., "Provably safe navigation for mobile robots with limited field-of-views in dynamic environments," $\mathrm{Au}$ tonomous Robots, vol. 32, no. 3, pp. 267-283, Apr. 2012. 